\title{
Estabelecimento de Echinochloa polystachya (H.B.K.) Hitchcock (Poaceae) em solo de várzea contaminado com petróleo de Urucu
}

\author{
Aline LOPES ${ }^{1}$, Maria Teresa Fernandez PIEDADE²
}

\begin{abstract}
RESUMO
A utilização de plantas para fitorremediação do solo requer que a concentração do poluente esteja dentro dos limites de tolerância da planta para que o tratamento seja eficaz. O rebrotamento e crescimento da herbácea aquática Echinochloa polystachya em solo de várzea contaminado por petróleo de Urucu foram estudados por meio do plantio de propágulos em frascos com $2 \mathrm{~L}$ de solo contaminado pela mistura de 7 dosagens de petróleo, distribuídas entre 0 e $0,46 \mathrm{~L} \mathrm{~m}^{-2}$. O experimento foi realizado em Casa de Vegetação e as plantas foram monitoradas durante 70 dias. Em resposta ao aumento da dosagem do petróleo, houve diminuição da biomassa aérea, da proporção de biomassa aérea viva/aérea total, do comprimento da folha e do número de folhas. A interação entre dosagem e tempo de exposição levou à diminuição do número de folhas, comprimento da folha, altura de inserção da primeira folha e aumento do número de folhas com comprometimento fitossanitário. A utilização de $E$. polystachya para fitorremediação pode estar limitada a solos com baixas dosagens de petróleo de Urucu.
\end{abstract}

PALAVRAS-CHAVE: Amazônia Central, Áreas alagáveis, Macrófitas aquáticas, Fitorremediação.

\section{Establishment of Echinochloa polystachya (HBK) Hitchcock in varzea soil contaminated with Urucu's petroleum}

\section{ABSTRACT}

The use of plants for the phytoremediation of contaminated soils requires that the concentrations of the pollutant lay within the limits of tolerance of the plant in order to ensure the efficacy of the treatment. The vegetative propagation and growth of the aquatic herbaceous plant Echinochloa polystachya were studied by planting parts of stems in $2 \mathrm{~L}$ of várzea soils previously contaminated by crude oil from Urucu, in 7 different dosages of oil ranging from 0 to $0.46 \mathrm{~L} \mathrm{~m}^{-2}$. The experiment took place in a green house and the plants were monitored during a 70 days period. Increasing the oil concentration lead to the decrease of the above-ground biomass, the ratio alive aerial biomass/total aerial biomass, leaf length and number of leaves. The interaction between dosage and time of exposition contributed for a reduction in the number and length of leaves, height of insertion of the first leaf, and increase of the number of unhealthy leaves. The use of E. polystachya for phytoremediation may be limited to soils with lower concentrations of crude oil from Urucu.

KEY wORDS: Central Amazon, Floodplains, Aquatic macrophytes, Phytoremediation.

1 Instituto Nacional de Pesquisas da Amazônia. Av. André Araújo, 2936, Aleixo, CEP 69060-001, Manaus - AM. E-mail: alopesmga@gmail.com

2 Instituto Nacional de Pesquisas da Amazônia, Coordenação de Pesquisas em Biologia Aquática. Av. André Araújo, 2936, Aleixo, CEP 69060-001, Manaus - AM. E-mail: maitepp@ inpa.gov.br 


\section{INTRODUÇÃo}

A exploração, transporte e armazenamento de petróleo na Bacia do Solimões (Amazonas - Brasil) são atividades que podem causar a contaminação das planícies de inundação (várzeas) ao longo desse rio. O solo contaminado por petróleo gera problemas ecotoxicológicos resultantes do potencial tóxico, carcinogênico e mutagênico dos hidrocarbonetos para as plantas, microrganismos (Pothuluri \& Cerniglia, 1994) e animais (Peterson et al., 2003). Petróleos leves como o de Urucu podem ser facilmente absorvidos pelas plantas, resultando em danos imediatos à vegetação (Pezeshki \& DeLaune, 1993) e esses danos podem se estender para outros grupos de organismos.

A recuperação de áreas contaminadas por atividades humanas pode ser feita por vários métodos, tais como escavação, incineração, extração com solventes, oxi-redução e outros que podem ser bastante dispendiosos. Alguns processos deslocam a matéria contaminada para locais distantes, causando riscos de contaminação secundária, aumentando ainda mais os custos do tratamento (Cunningham et al., 1995), e causando mais danos à vegetação, devido à necessidade de tráfego de máquinas e o constante pisoteio humano (Pezeshki et al., 2000). Por isso, atualmente tem-se dado preferência a métodos que perturbem menos o ambiente e sejam mais econômicos. Nesse contexto, a fitorremediação é uma alternativa capaz de empregar sistemas vegetais fotossintetizantes e sua microbiota associada com a finalidade de desintoxicar ambientes degradados ou poluídos (Salt et al., 1995; Siciliano \& Germida, 1999).

A fitorremediação oferece várias vantagens que devem ser consideradas, como a capacidade de tratar grandes áreas contaminadas por diversos poluentes, a baixo custo, com possibilidades de remediar águas contaminadas, o solo e o subsolo. Para isso, a concentração do poluente e a presença de toxinas devem estar dentro dos limites de tolerância da planta usada para assegurar a eficácia do tratamento. Os riscos de que os vegetais venham a entrar na cadeia alimentar devem ser considerados ao empregar esta tecnologia. Além disso, o tempo para se obter resultados satisfatórios pode ser longo (Cunningham et al., 1995). O sucesso do tratamento empregando plantas aquáticas reside nas inúmeras possibilidades de reciclagem da biomassa produzida. Conforme a toxicidade da biomassa esta pode ser utilizada como fertilizante, ração animal, para a geração de energia (biogás ou queima direta), fabricação de papel, extração de proteínas para uso em rações, extração de substâncias quimicamente ativas de suas raízes para uso como estimulante de crescimento de plantas, entre outros (Glass, 1998).

As substâncias alvo da fitorremediação incluem metais, compostos inorgânicos, elementos químicos radioativos, hidrocarbonetos derivados do petróleo, pesticidas, herbicidas, solventes clorados, resíduos orgânicos industriais, entre outros
(Cunningham et al., 1995). No caso da contaminação do solo por petróleo, acredita-se que os efeitos da fitorremediação ocorram pela estimulação da capacidade de degradação dos microrganismos da rizosfera, denominada fitoestimulação ou rizo-biodegradação (Anderson et al., 1993). As gramíneas são comumente utilizadas para a fitorremediação devido ao seu crescimento rápido e pela sua extensiva ramificação radicular que proporciona uma ampla superfície, facilitando a interação planta-microbiota (Hutchinson et al., 2001; Glick, 2003).

Entre as herbáceas aquáticas de importância nas várzeas amazônicas, destaca-se a gramínea Echinochloa polystachya. A espécie forma grandes bancos homogêneos em águas ricas em nutrientes (Piedade et al., 1991, Piedade, 1993). Alguns estudos realizados com E. polystachya no México, em solos contaminados com hidrocarbonetos de petróleo intemperizados, mostram que a planta é capaz de sobreviver nestes ambientes, embora possa apresentar uma redução de até 53\% em sua biomassa (Rivera-Cruz et al., 2001, 2004). A espécie também tem se mostrado capaz de acumular metais pesados em suas raízes e folhas, sem sofrer grandes alteraçōes no crescimento quando exposta a altas concentraçôes de cádmio (até 174 mg L-1 $\mathrm{Cd}^{2+}$; Solís-Domínguez et al., 2007), sugerindo que seria uma espécie promissora para restauração de ambientes contaminados com petróleo.

A utilização de uma planta nativa e com alta capacidade reprodutiva como $E$. polystachya para fitorremediação pode ser uma alternativa adequada e importante para a recuperação de solos de várzea na Amazônia. Entretanto, para que haja eficácia na fitorremediação de uma área é necessária a realização de estudos prévios acerca da toxicidade dos poluentes sobre as plantas, porque as vantagens da fitorremediação podem ser neutralizadas pelo efeito tóxico do contaminante, alterando não somente o acúmulo de biomassa como também a morfologia das plantas (Merlk et al., 2005). O objetivo deste estudo foi avaliar a capacidade de rebrotamento e de crescimento de propágulos de Echinochloa polystachya visando determinar a potencialidade deste vegetal para o uso na fitorremediação de solo de várzea contaminado por petróleo de Urucu.

\section{MATERIAIS E MÉTODOS}

\section{ÁREA DE ESTUDO}

A macrófita aquática perene Echinochloa polystachya foi coletada em setembro de 2006 na Ilha da Marchantaria $\left(03^{\circ} .15^{\prime} \mathrm{S}, 060^{\circ} .00^{\prime} \mathrm{W}\right)$, na várzea da Amazônia Central, Amazonas, Brasil.

O experimento foi realizado na Casa de Vegetação do Projeto INPA/Max-Planck, Manaus, Amazonas, Brasil. As plantas foram irrigadas diariamente com água proveniente do poço artesiano. O solo utilizado no plantio dos propágulos 
foi coletado no mesmo local e no mesmo período da coleta das plantas.

\section{MATERIAL VEGETAL}

Echinochloa polystachya (Poaceae) é um capim semiaquático, emergente, perene, amplamente distribuído na planície de inundação do Rio Amazonas. O ciclo de vida da planta é fortemente relacionado ao ciclo hidrológico, e inclui uma rebrota, quando o nível da água é baixo, na fase terrestre, a partir dos nós presentes nos talos da velha geração de plantas. A rebrota ocorre quando as chuvas se intensificam. Em condiçôes ambientais favoráveis, a espécie chega a atingir $80 \mathrm{t} \mathrm{ha}^{-1}$ de biomassa, no pico da inundação do rio Amazonas, e uma produtividade primária em torno de $108 \mathrm{t} \mathrm{ha}^{-1}$ ano $^{-1}$ (Piedade et al. 1991), em um ciclo anual de crescimento. A produtividade da espécie está acima da faixa sugerida para macrófitas aquáticas $\left(8 \mathrm{a} 60 \mathrm{t} \mathrm{ha}^{-1}\right)$, sendo 4 vezes maior que a sugerida para florestas tropicais chuvosas (Lieth, 1975). Sua biomassa está distribuída em 65\% de caules, $30 \%$ de folhas e $5 \%$ de raízes (Piedade, 1988). Essa biomassa é consumida por capivaras e algumas tartarugas; as folhas servem à alimentação de invertebrados terrestres e as raízes proporcionam substrato para invertebrados aquáticos e hábitat para peixes jovens (Piedade et al., 1992). Devido aos altos valores de produtividade primária produzida pela espécie ela é considerada fundamental para o ecossistema de várzea. Estudos prévios mostram que a concentração de elementos químicos é muito semelhante entre as diferentes partes do colmo e entre os períodos do ano, de tal forma que cada propágulo vegetativo tem porçôes adequadas e equiparáveis de nutrientes para o seu rebrotamento (Piedade et al., 1992; Piedade et al., 1997).

\section{PETRÓLEO}

Foi utilizado no experimento petróleo proveniente da Base Petrolífera de Urucu, Amazonas, Brasil. Este petróleo é considerado leve e de alta qualidade, com grau API de 46,8 (ANP, 2008). As dosagens de petróleo foram escolhidas por meio da realização de um estudo prévio com dosagens agudas de até 15,9 $\mathrm{L} \mathrm{m}^{-2}$ de solo (Lopes et al., 2005).

\section{DESENHO EXPERIMENTAL}

Foram coletadas plantas adultas, das quais foi cortado um colmo de $15 \mathrm{~cm}$ contendo um nó. Estes propágulos foram plantados em bandejas plásticas (27,0 cm de largura, 40,0 de comprimento e 7,0 cm de profundidade) com 2 litros de solo da várzea previamente contaminado pela adição de petróleo. O petróleo foi misturado ao volume total de solo para simular o plantio em uma área contaminada, onde geralmente ocorre o revolvimento do solo, e para que ficasse à disposição da plântula via captação pela raiz em qualquer parte do substrato (OECD, 2006). As dosagens aplicadas foram 0 (controle),
1,5; 3; 6; 12,5; 25 e $50 \mathrm{~mL}$ em 2L de solo, com 10 réplicas para cada tratamento (Tabela 1 ).

A cada sete dias foram monitorados o surgimento de brotos e o número destes em cada unidade amostral, pois esta espécie possui a capacidade de produzir um ou mais brotos por nó (Piedade, 1993). Foi contado ainda o número de folhas normais e com comprometimento fitossanitário (folhas com menos de $30 \%$ da área verde) e medido o comprimento das folhas e a altura de inserção da primeira folha. Após 70 dias as plantas foram cuidadosamente retiradas dos vasos, lavadas e separadas em biomassa aérea viva, biomassa aérea morta, biomassa de raízes e biomassa do colmo, para obtenção da biomassa total (peso seco). A biomassa total foi calculada utilizando a seguinte equação: $\mathrm{Bt}=\mathrm{ba}+\mathrm{bb}+\mathrm{bc}$. Onde: $\mathrm{Bt}=$ biomassa total; $\mathrm{ba}=$ biomassa aérea viva; $\mathrm{bb}=$ biomassa aérea morta; $\mathrm{bc}=$ biomassa das raízes. Como o efeito da dosagem do petróleo sobre a biomassa total muitas vezes é mascarado porque enquanto a biomassa viva diminui a biomassa morta aumenta, alguns autores utilizam a proporção biomassa aérea viva/biomassa aérea total para avaliar o efeito do petróleo sobre a biomassa das plantas (Lin \& Mendelssohn, 1996).

Tabela 1 - Dosagens de petróleo aplicadas baseadas no volume por unidade amostral, volume por unidade de área superficial, peso do solo seco e a porcentagem de óleo em relação ao volume de solo.

\begin{tabular}{|c|c|c|c|}
\hline $\begin{array}{l}\mathrm{mL} \text { óleo por } 2 \mathrm{~L} \\
\text { de solo }\end{array}$ & $\begin{array}{l}\text { L óleo } \mathrm{m}^{-2} \mathrm{de} \\
\text { superfície }\end{array}$ & $\begin{array}{l}\text { mg de óleo } \mathrm{g}^{-1} \text { de } \\
\text { solo seco }\end{array}$ & $\%(g / g)$ \\
\hline 0 & 0 & 0 & 0 \\
\hline 1,5 & 0,014 & 0,55 & 0,0006 \\
\hline 3,0 & 0,028 & 1,09 & 0,0011 \\
\hline 6,0 & 0,056 & 2,28 & 0,0023 \\
\hline 12,5 & 0,116 & 4,56 & 0,0047 \\
\hline 25,0 & 0,231 & 9,12 & 0,0094 \\
\hline 50,0 & 0,463 & 18,24 & 0,0188 \\
\hline
\end{tabular}

\section{ANÁLISE ESTATÍSTICA}

As análises estatísticas foram realizadas utilizando o programa SYSTAT 10.2 (Systat Inc., 2002). Os efeitos do tempo e da dosagem de petróleo sobre as variáveis dependentes foram avaliados por meio de Análise de Variância bifatorial com medidas repetidas (ANOVAmr), respeitando-se os pressupostos de homocedasticidade e esfericidade. Para estabelecer o efeito do petróleo sobre a biomassa foi realizada Análise de Variância unifatorial, e o teste de Tukey foi utilizado para avaliar diferenças estatísticas individuais entre os níveis dos tratamentos. As diferenças significativas são relatadas no nível de 0,05 de probabilidade, a menos que indicadas de outra maneira. 


\section{RESULTADOS}

Houve um aumento significativo do número de brotos por unidade amostral ao longo do tempo (Figura 1a). Porém, foi verificada uma diminuição do número médio de brotos por unidade amostral (Tabela 2) devido ao aumento da dosagem de petróleo. O número de rebrotamentos por unidade amostral permaneceu praticamente constante após o $21^{\circ}$ dia de tratamento. As diferenças a partir desta data se deveram, principalmente, à morte de brotos que não se desenvolveram nos potes onde havia ocorrido o rebrotamento de mais de uma plântula por nó. Ao término do experimento dez propágulos não haviam rebrotado, sendo que cinco destes eram do tratamento com $0,46 \mathrm{~L} \mathrm{~m}^{-2}$ de petróleo, quatro no tratamento de $0,23 \mathrm{~L} \mathrm{~m}^{-2}$ e um no tratamento com $0,05 \mathrm{~L} \mathrm{~m}^{-2}$. Apenas cinco dos potes restantes apresentavam mais de um broto.
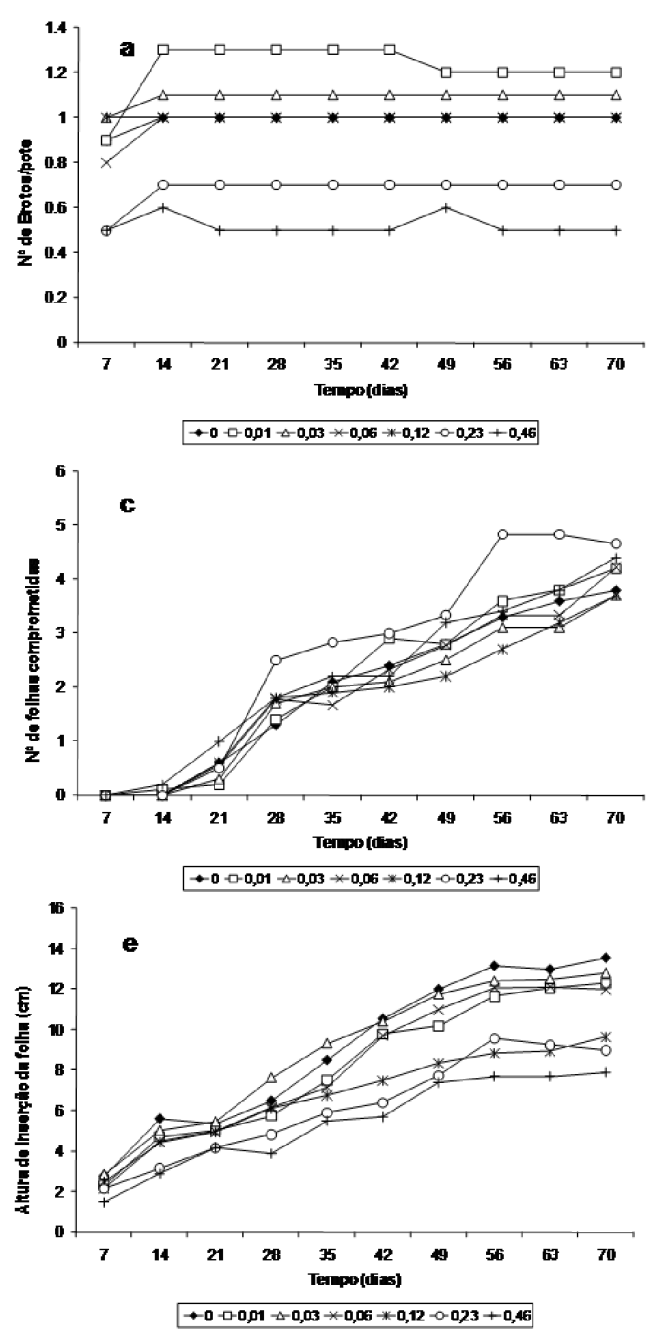

$\mathrm{O}$ incremento do número de folhas de E. polystachya foi afetado significativamente pelo tempo de exposição (Tabela 2). Ao longo do tempo de exposição o aumento do número de folhas nos tratamentos com maiores dosagens foi decaindo e nos de menores dosagens aumentando, o que resultou em uma interação significativa entre o tempo de exposição e dosagem de petróleo (Figura 1b). O inverso foi observado para o incremento do número de folhas com comprometimento fitossanitário no qual o efeito de interação entre o tempo de exposição e a dosagem de petróleo causou um aumento maior de folhas comprometidas nos tratamentos de dosagens mais elevadas, após 56 dias de tratamento (Figura 1c). Neste caso, o tempo de exposição também causou um aumento do número de folhas com comprometimento fitossanitário (Tabela 2). Apesar do aumento gradual do número de folhas comprometidas à medida que a dosagem do petróleo aumentou, o tratamento
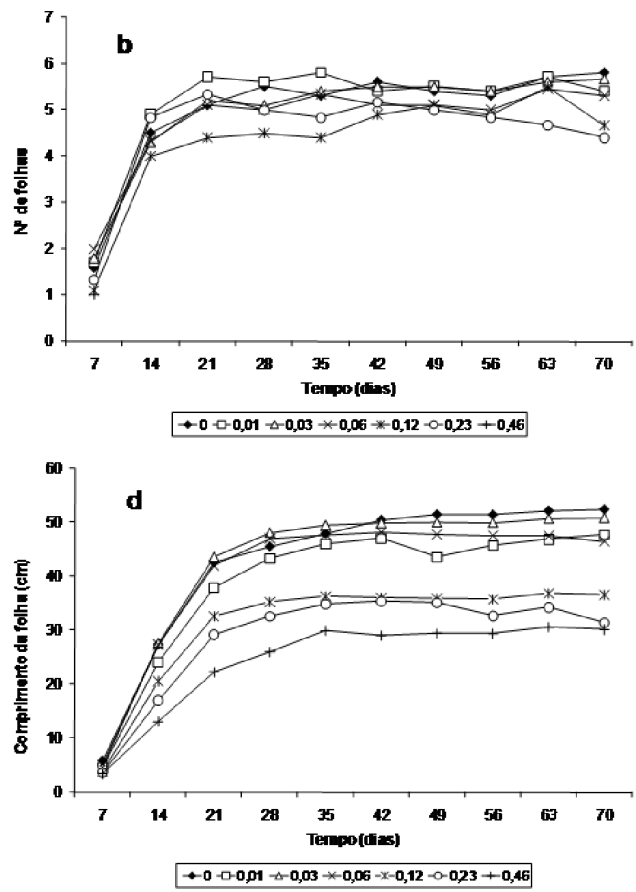

Figura 1 - Efeito da dosagem de petróleo de Urucu $\left(\mathrm{L} \mathrm{m}^{-2}\right)$ sobre Echinochloa polystachya ao longo do tempo de exposição, nos valores médios de: a) número de brotos $(\mathrm{dp}<0,7)$; b) número de folhas (dp<1,5); c) número de folhas com comprometimento fitossanitário $(\mathrm{dp}<1,5)$; $\mathrm{d}$ ) comprimento da folha $(\mathrm{dp}<11,9)$; e) altura de inserção da primeira folha $(\mathrm{dp}<3,6)$. dp = desvio-padrão . 
de $0,23 \mathrm{~L} \mathrm{~m}^{-2}$ apresentou um aumento mais acentuado que os demais (Figura 1c). Isso pode ter ocorrido porque as plantas do tratamento de $0,46 \mathrm{~L} \mathrm{~m}^{-2}$ eram menores e apresentavam menos folhas e, consequentemente, esta situação limitou o número de folhas comprometidas.

O tempo causou um aumento significativo $(p<0,0001)$ da média do comprimento das folhas em todos os tratamentos (Tabela 2), tendo sido atingido um comprimento máximo no tratamento de $0,03 \mathrm{~L} \mathrm{~m}^{-2}$ de petróleo de $74,5 \mathrm{~cm} \mathrm{e} 68,0 \mathrm{~cm}$ no controle, enquanto que no tratamento de $0,46 \mathrm{~L} \mathrm{~m}^{-2}$ o comprimento máximo foi de $38,5 \mathrm{~cm}$. Houve, ainda, a formação de dois padrôes de aumento do comprimento das folhas, 0 a $0,05 \mathrm{~L} \mathrm{~m}^{-2}$ e 0,12 a $0,23 \mathrm{~L} \mathrm{~m}^{-2}$, resultando em uma interação significativa entre a dosagem de petróleo e o tempo de exposição (Figura 1d). Este mesmo padrão foi observado para a altura da inserção da primeira folha (Figura 1e), indicando que dosagens acima de $0,12 \mathrm{~L} \mathrm{~m}^{-2}$ são prejudiciais ao desenvolvimento das plantas.

Tabela 2 - Resultados da ANOVAmr para verificar o efeito da dosagem de petróleo (D) e do tempo de exposição (T) em E. polystachya, sobre as variáveis avaliadas. $T * D$ (interação); g.l. (graus de liberdade).

\begin{tabular}{|c|c|c|c|c|c|}
\hline Variável dependente & Fator & $\begin{array}{l}\text { g.l. } \\
\text { efeito }\end{array}$ & g.l. erro & $\mathrm{F}$ & $p$ \\
\hline \multirow{3}{*}{$\mathrm{N}^{0}$ de brotos/pote } & D & 6 & 63 & 3,47 & 0,005 \\
\hline & $\mathrm{T}$ & 8 & 504 & 4,59 & $<0,001$ \\
\hline & $T * D$ & 48 & 504 & 0,79 & 0,837 \\
\hline \multirow{3}{*}{$N^{0}$ de folhas } & $\mathrm{D}$ & 6 & 53 & 0,93 & 0,475 \\
\hline & $\mathrm{T}$ & 8 & 424 & 15,11 & $<0,001$ \\
\hline & $T^{*} \mathrm{D}$ & 48 & 424 & 1,53 & 0,016 \\
\hline \multirow{3}{*}{$\begin{array}{l}N^{0} \text { de folhas com } \\
\text { comprometimento } \\
\text { fitossanitário** }\end{array}$} & $\mathrm{D}$ & 6 & 53 & 1,30 & 0,272 \\
\hline & $\mathrm{T}$ & 7 & 371 & 190,21 & $<0,001$ \\
\hline & $T * D$ & 42 & 371 & 1,46 & 0,035 \\
\hline \multirow{3}{*}{$\begin{array}{l}\text { Comprimento das } \\
\text { folhas }\end{array}$} & $\mathrm{D}$ & 6 & 53 & 5,76 & $<0,001$ \\
\hline & $\mathrm{T}$ & 8 & 424 & 455,31 & $<0,001$ \\
\hline & $T^{*} \mathrm{D}$ & 48 & 424 & 2,73 & $<0,001$ \\
\hline \multirow{3}{*}{$\begin{array}{l}\text { Altura de inserção da } \\
\text { primeira da folha }\end{array}$} & $\mathrm{D}$ & 6 & 53 & 3,96 & 0,002 \\
\hline & $\mathrm{T}$ & 8 & 424 & 242,70 & $<0,001$ \\
\hline & $T * D$ & 48 & 424 & 2,40 & $<0,001$ \\
\hline
\end{tabular}

** foram utilizados os dados a partir do $14^{\circ}$ dia de tratamento, devido a baixa ocorrência de folhas com problemas sanitários.

** data were used from the $14^{\circ}$ day of treatment, due to the lower occurrence of leaves with sanitary problems.

A biomassa aérea das plantas foi afetada pelo petróleo, havendo redução com o aumento da dosagem $\left(\mathrm{F}_{(6,53)}=6,28\right.$; $\mathrm{p}<0,0001)$. Diferenças significativas em relação ao controle foram encontradas nos tratamento a partir de $0,12 \mathrm{~L} \mathrm{~m}^{-2}$ (Figura 2a). A redução da biomassa aérea foi gradual com o aumento da dosagem de petróleo, apresentando uma média de $1,24 \pm 0,43 \mathrm{~g}$ no tratamento controle, $0,68 \pm 0,233 \mathrm{~g}$ no tratamento de $0,12 \mathrm{~L} \mathrm{~m}^{-2}$ e $0,42 \mathrm{~g} \pm 0,29 \mathrm{~g}$ no tratamento com $0,46 \mathrm{~L} \mathrm{~m}^{-2}$. Não houve efeito da dosagem de petróleo sobre a produção de biomassa aérea morta, biomassa do colmo e biomassa das raízes ( $p>0,05)$, porém, houve uma tendência de efeito da dosagem sobre a biomassa total $\left(\mathrm{F}_{(6,53)}\right.$ $=2,22, \mathrm{p}=0,056)$. Contudo, quando avaliada a proporção de biomassa aérea viva/biomassa aérea total houve uma redução significativa $\left(\mathrm{F}_{(6,53)}=5,61 ; \mathrm{p}<0,0001\right)$ em relação ao controle, nos tratamentos de 0,23 e $0,46 \mathrm{~L} \mathrm{~m}^{-2}$ (Figura $2 \mathrm{~b}$ ).
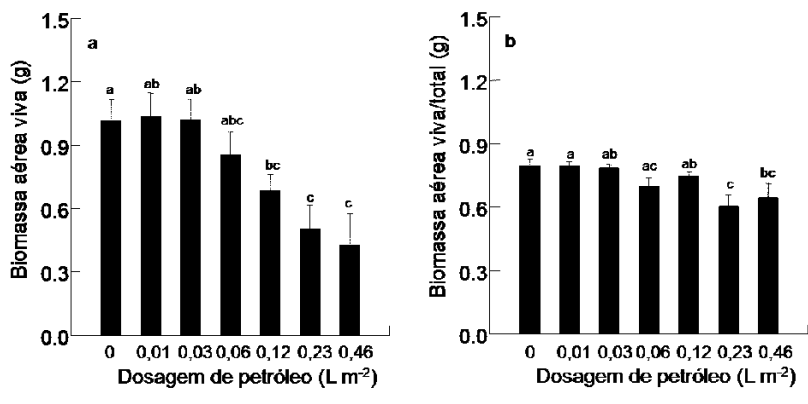

Figura 2 - Efeito do petróleo de Urucu sobre: a) a biomassa aérea; b) a proporção de biomassa aérea viva/ aérea total de Echinochloa polystachya. Valores médios com erro padrão. Médias com letras iguais não são significativamente diferentes.

\section{DISCUSSÃO}

A dosagem de petróleo e o tempo de exposição influenciaram a resposta de Echinochloa polystachya quando plantada em solo de várzea contaminado por petróleo de Urucu. Os efeitos dos hidrocarbonetos do petróleo na vegetação dependem geralmente do volume do óleo, da sua concentração no solo e da quantidade que entrou em contato com esta (Lin \& Mendelssohn, 1996; Pezeshki et al., 2000). No presente estudo o aumento da concentração de hidrocarbonetos afetou a capacidade de estabelecimento e desenvolvimento das plantas, podendo impossibilitar tentativas de fitorremediação com esta espécie em ambientes contaminados com dosagens elevadas de petróleo. Por outro lado, como E. polystachya é uma espécie abundante na planície de inundação do Rio Amazonas, alteraçôes em sua densidade poderiam ser um indicativo e servir como um instrumento para o acompanhamento da integridade ambiental das áreas susceptíveis a vazamentos.

Foram observadas diversas alterações no desenvolvimento das plântulas devido à exposição ao petróleo. Dentre os efeitos mais nocivos está a redução da capacidade de rebrotamento da espécie proporcionalmente ao aumento da dosagem de petróleo, visto que no ambiente natural esta é a principal forma de reprodução desta espécie (Piedade et al., 1992). Os indivíduos que rebrotaram em solo contaminado sofreram diversos danos no crescimento, com redução do número, comprimento e altura da inserção das folhas, e aumento no incremento de folhas com comprometimento fitossanitário. Como E. polystachya é uma espécie de extrema importância para o ecossistema de várzea, devido aos altos valores de 
produtividade primária anual produzida e por disponibilizar um importante substrato para diversos organismos da biota aquática (Piedade et al., 1991), a redução na densidade de suas populaçōes pode gerar uma cascata de efeitos nocivos indiretos diminuindo a produtividade geral do sistema.

Os resultados do presente estudo quando comparados aos de Lopes (2007), que analisou a mortalidade de E. polystachya resultante da exposição ao petróleo de Urucu durante 20 dias em indivíduos juvenis (30 dias de plantio), mostram que a espécie foi mais sensível ao petróleo nas fases iniciais do rebrotamento e desenvolvimento dos propágulos que na fase juvenil. Apesar de E. polystachya ter sido capaz de rebrotar em solo contaminado com até $0,46 \mathrm{~L} \mathrm{~m}^{-2}$ solo as plântulas sofreram redução nos diversos parâmetros biométricos analisados (número de folhas, comprimento das folhas, biomassa aérea, número de folhas com comprometimento fitossanitário) em dosagens acima de $0,05 \mathrm{~L} \mathrm{~m}^{-2}$ solo, enquanto que os juvenis só apresentaram alterações dos mesmos parâmetros em dosagens acima $0,23 \mathrm{~L} \mathrm{~m}^{-2}$ solo. Isto reforça a afirmação de Pezeshki et al. (2000) de que as plantas são mais sensíveis ao óleo no período de crescimento que durante a outras fases da vida. Na estação de crescimento os processos metabólicos nas plantas são intensos e fundamentais para o desenvolvimento do indivíduo adulto e, sob tais circunstâncias, o estresse da exposição ao petróleo pode levar à interrupção ou ao comprometimento irreversível de funções fisiológicas vitais (Pezeshki et al., 2000).

Comparações entre os resultados obtidos com $E$. polystachya e com outras espécies são limitados já que os efeitos do petróleo variam com o tipo do óleo, com a dosagem, e com a espécie testada (Lin \& Mendelssohn, 1996; Pezeshki et al., 2000; Lin et al., 2002). Além disso, estudos toxicológicos em ecossistemas alagáveis em regiōes tropicais ainda são escassos. Os poucos estudos realizados com a utilização de petróleo de Urucu mostram a alta toxicidade deste petróleo sobre as plantas aquáticas. Crema (2003) observou que a dosagem de $3 \mathrm{~L} \mathrm{~m}^{-2}$ de água provocou a morte de Eichhornia crassipes. A exposição de Pistia stratiotes por 98 dias a $0,3 \mathrm{~L} \mathrm{~m}^{-2}$ de água causou a mortalidade de todos os indivíduos (Silva \& Camargo, 2007). No entanto, Victório et al. (2006) encontraram aumento de biomassa total após a exposição por 20 dias de Egeria densa a dosagens de petróleo de $0,06 \mathrm{~L} \mathrm{~m}^{-2}$ e $0,12 \mathrm{~L} \mathrm{~m}^{-2}$ de água, sugerindo que essa planta é beneficiada pela exposição ao petróleo. Por ser de hábito submerso enraizado, $E$. densa não teve contato direto com o petróleo, que formou uma lâmina na superfície da água, porém foi beneficiada pelo enriquecimento do meio com carbono inorgânico.

Quando comparado o efeito do petróleo de Urucu com o de outros tipos de petróleo, percebe-se a alta toxicidade desse óleo para as plantas. Espécies como Spartina alterniflora e Spartina patens quando expostas a petróleo pesado como o do sul da Louisiana só apresentaram inibição de crescimento em dosagens superiores a $8 \mathrm{~L} \mathrm{~m}^{-2}$ solo (Lin \& Mendelssohn, 1996) quantidade bem acima da utilizada neste experimento $\left(0,46 \mathrm{~L} \mathrm{~m}^{-2}\right)$. Porém, S. alterniflora quando exposta ao óleo combustível No 2 (leve), sofreu redução da biomassa em dosagens acima de $2,5 \mathrm{~L} \mathrm{~m}^{-2}$ de solo. Estas diferenças se devem dentre outros fatores a densidade do petróleo. $\mathrm{O}$ petróleo de Urucu é considerado leve porque contem $15 \%$ de hidrocarbonetos aromáticos (pesados), 20,2\% de compostos naftalênicos (médios) e 64,8\% de compostos parafínicos (leves) (Petrobrás, 1997). Os óleos leves atuam em nível celular, alterando a permeabilidade da membrana ou interrompendo vários processos do metabolismo da planta e seus efeitos parecem ser imediatamente tóxicos às plantas, enquanto que óleos mais pesados causam danos físicos, provocando asfixia e impedindo a troca de gases (Pezeshki et al., 2000).

Estudos toxicológicos demonstraram não haver diferenças significativas na biomassa de E. polystahya quando exposta a altas concentrações de metais pesados (Solís-Domínguez et. al., 2007), ou a hidrocarbonetos policíclicos aromáticos (benzo[a] pireno em dosagens de até $100 \mathrm{mg} \mathrm{kg}^{-1}$ ) presentes no petróleo (Alarcón et al., 2006). Já em solos contaminados com hidrocarbonetos de petróleo (HTP) intemperizados, $E$. polystachya foi capaz de sobreviver, embora apresentasse uma redução de até 53\% em sua biomassa em áreas com 79,457 mg. $\mathrm{kg}^{-1}$ de HTP em um período de 150 dias de avaliação (Rivera-Cruz et al., 2004). No presente estudo a redução da biomassa aérea foi maior, da ordem de $65,5 \%$ no tratamento com $0,46 \mathrm{~L} \mathrm{~m}^{-2}$, confirmando a alta toxicidade do petróleo de Urucu.

O monitoramento do impacto do petróleo sobre $E$. polystachya durante 70 dias permitiu confirmar que a duração do tempo de exposição é um fator determinante na maximização dos efeitos do petróleo de Urucu sobre esta espécie. Isto pôde ser constatado pela diminuição do comprimento das folhas e altura de inserção da primeira folha que não haviam sido alterados pela exposição por 20 dias em dosagens de até 15,89 L do petróleo de Urucu $\mathrm{m}^{2}$ solo (Lopes, 2007). Projetos de revegetação de áreas contaminadas com a utilização de $E$. polystachya podem ser estabelecidos desde que considerados os efeitos do petróleo em curto e longo prazos, bem como os riscos de incorporação de material contaminado à cadeia alimentar.

\section{CONCLUSÃO}

Doses mais altas do petróleo, como $0,46 \mathrm{~L} \mathrm{~m}^{-2}$ solo foram limitantes para o estabelecimento de E. polystachya, o que pode ser constatado pelo reduzido número de rebrotamento neste tratamento. O tempo de exposição foi um fator agravante do impacto, e produziu efeito sobre todos os parâmetros avaliados. Pode-se sugerir a partir desses resultados, que $E$. 
polystachya é capaz de se desenvolver em solos contaminados com baixas quantidades de petróleo de Urucu, ou seja, menores que $0,12 \mathrm{~L} \mathrm{~m}^{-2}$ solo. Por outro lado, experimentos em campo devem ser delineados para analisar a capacidade desta planta de reduzir os componentes tóxicos do petróleo e assim poder ser efetivamente utilizada na fitorremediação.

\section{AGRADECIMENTOS}

Os recursos necessários para este estudo foram providos pelo Projeto INPA/Max-Planck, PPI 1090-5, e o petróleo cedido pela Petrobrás através do Projeto PIATAM. Agradecemos ao $\mathrm{CNPq}$ pela Bolsa de Mestrado e ao Programa BECA - IEB/Fundação Moore (B/2007/01/DEP/15) pela Bolsa de Desenvolvimento Profissional cedidas à Aline Lopes. Agradecemos aos três revisores anônimos pelas valiosas sugestôes que enriqueceram muito o trabalho. Agradecemos aos técnicos, Celso Rabelo Costa e Valdeney de Araújo Azevedo pelo auxílio na coleta dos dados.

\section{BIBLIOGRAFIA CITADA}

Alarcón, A.; Delgadillo-Martínez, J.; Franco-Ramírez, A.; Davies, F.T.Jr.; Ferrera-Cerrato, R. 2006. Influence of two polycyclic aromatic hydrocarbons on spore germination, and phytoremediation potential of Gigaspora margarita-Echynochloa polystachya symbiosis in benzo(a)pyrene-polluted substrate. Revista Internacional de Contaminación Ambiental, 22: 39-47.

ANP - Agência Nacional do Petróleo, Gás Natural e Biocombustíveis. 2008. Despacho do Diretor-Geral no 683, Anexo II: relação de tipos de petróleo nacional, pp.13. (http://www.anp.gov.br/doc/ participacoes_governamentais/2008/Revisao_2008.pdf) Acesso: 18/02/2009.

Anderson, T.A.; Guthrie, E.A.; Walton, B.T. 1993. Bioremediation in the rhizosphere: plant roots and associated microbes clean contaminated soil. Environmental Science Technology, 27: 26302636.

Crema L.C. 2003. Efeito de diferentes concentrações de petróleo sobre o crescimento da macrófita aquática Eichhornia crassipes. Trabalho de Conclusão de Curso, Instituto de Biociências, Universidade Estadual Paulista, 16pp.

Cunningham, S.D.; Berti, W.R.; Huang, J.W. 1995. Phytoremediation of contaminated soils. Trends in Biotechnology, 13: 393-397.

Glass, D.J. 1998. The 1998 United States Market for Phytoremediation, D. Glass Associates, Needham, 139pp.

Glick, B.R. 2003. Phytoremediation: synergistic use of plants and bacteria to clean up the environment. Biotechnological Advances, 21: 383-393.

Hutchinson, S.L.; Banks, M.K.; Shwab, A.P. 2001. Phytoremediation of age petroleum sluge: effect of inorganic fertilizer. Journal of Environmetal Quality, 30: 395-403.

Lieth, H. 1975. Primary production of the major vegetation units of the word. In: Lieth, H.; Whittaker, R. H. (Eds.) Primary Productivity of the Biosphere, Springer - Verlag, New York, p. 203-215.
Lin, Q.; Mendelssohn, I.A. 1996. A comparative investigation of the effects of Louisiana crude oil on the vegetation of fresh, brackish, and salt marsh. Marine Pollution Bulletin, 32 (2): 202-209.

Lin, Q.; Mendelssohn, I. A.; Suidam, M. T.; Lee, K.; Venosa, A.D. 2002. The dose-response relationship between No. 2 fuel oil and the growth of the salt marsh grass, Spartina alterniflora. Marine Pollution Bulletin, 44: 897-902.

Lopes, A. 2007. Respostas de Herbáceas Aquáticas Amazônicas ao Petróleo Cru de Urucu (Coari-AM). Dissertação de Mestrado, Instituto Nacional de Pesquisas da Amazônia/Universidade Federal do Amazonas, Manaus, Amazonas. 129pp.

Lopes, A.; Piedade, M.T.F.; Almeida-Val, V.M.F. 2005. Efeito da dosagem do petróleo de Urucu (Coari-AM) sobre a sobrevivência de Echinochloa polystachya (Projeto Piloto), Anais do I Congresso Internacional do PIATAM. Universidade Federal do Amazonas / Centro de Pesquisas da Petrobrás, Manaus, Amazonas, 113113.

Merkl, N.; Schltze-Kraft, R.; Infante, C. 2005. Phytoremediation in the tropics - influence of heavy crude oil on root morphological characteristics of graminoids. Environment Pollution, 138: 86-91.

OECD, 2006. Test No. 208: Terrestrial Plant Test: Seedling Emergence and Seedling Growth Test. OECD guidelines for the testing of chemicals ENV/JM/TG(2005)4. (http://puck.sourceoecd.org/ $\mathrm{vl}=8550520 / \mathrm{cl}=43 / \mathrm{nw}=1 / \mathrm{rpsv} / \mathrm{ij} /$ oecdjournals $/ 1607310 \mathrm{x} / \mathrm{v} 1 \mathrm{n} 2 /$ s9/p1) Acesso em 17/03/2009.

Peterson, C.H.; Rice, S.D.; Short, J.W.; Esler, D.; Bodkin, J.L.; Ballachey, B.E.; Irons, D.B. 2003. Long-Term Ecosystem Response to the Exxon Valdez Oil Spill. Science, 302: 20822086.

Pezeshki, S.R; DeLaune, R.D. 1993. Effect of crude oil on gas exchange functions of Juncus roemerianus and Spartina alterniflora. Water, Air, and Soil Pollution, 68: 461-468.

Pezeshki, S.R.; Hester, M.W.; Lin, Q.; Nyman, J.A. 2000. The effect of oil spill and cleanup on dominant US Gulf coast marsh macrophytes: a review. Environment Pollution, 180: 129-139.

Petrobras. 1997. Caracteristicas do petróleo Urucu. CNPES:SEPESQ:DIQUIM:SETAV Anexo V.

Piedade, M.T.F. 1988. Biomassa, produtividade e atividade fotossintética de Echinochloa polystachya (H.B.K.) Hitchcock (Gramineae $=$ Poacea), capim semi-aquático da várzea amazônica . Tese de Doutorado. Instituto Nacional de Pesquisas da Amazônia/Universidade Federal do Amazonas, Manaus, Amazonas. 154pp.

Piedade, M.T.F. 1993. Biologia e ecologia de Echinochloa polystachya (H.B.K) Hitchcock (Gramineae=Poaceae), capim semi-aquatico da várzea amazônica. Acta Limnologica Brasiliensis, 4: 173-185.

Piedade, M.T.F.; Junk, W.J.; Long, S.P. 1991. The productivity of the C4 grass Echinochloa polystachya on the Amazon floodplain. Ecology, 72(4): 1456-1463.

Piedade, M.T.F.; Junk, W. J.; Mello, J.A.S.N. 1992. A floodplain grassland of the Central Amazon. In: Long, S. P.; Jones, M. B.; Roberts, J. M. (Eds.). Productivity of grass ecosystems of the Tropics and Sub-Tropics. Chapman, Hall/UNEP, London, p. 127-158. 
Piedade, M.T.F.; Junk, W.J.; Long, S.P. 1997. Nutrients dynamics of the highly productive C4 macrophyte Echinochloa polystachya on the Amazon floodplain. Functional Ecology, 11: 60-65.

Pothuluri, V.J.; Cerniglia, E.C. 1994. Microbial metabolismo f polycyclic aromatic hydrocarbons. In: R.G Chaudry (Ed.). Biological Degradation and Biorremediation of Toxic Chemicals. Dioscorides, Portland. p. 92-123.

Salt, D.E.; Blaylock, M.; Kumar, N.P.B.A.; Dushenkov V.; Ensley, B.D.; Chet I.; Raskin, I. 1995. Phytoremediation: a novel strategy for the removal of toxic metals from the environment using plants. Biotechnology, 13:468-474.

Siciliano, S. D.; Germida, J.J. 1999. Enhanced phytoremediation of chlorobenzoates in rhizospere soil. Soil Biology and Biochemistry, 31: 299-305.

Silva, R.S.; Camargo, A.F.M. 2007. Effect of Urucu crude oil on the aquatic macrophyte Pistia stratiotes. Acta Limnologica Brasiliensia, 19(2): 155-161.

Solis-Dominguez, F.A.; Gonzalez-Chavez, M.C.; Carrillo-Gonzalez, R.; Rodriguez-Vazquez, R. 2007. Accumulation and localization of cadmium in Echinochloa polystachya grown within a hydroponic system. Journal of Hazardous Materials, 141(3): 630-636.

Rivera-Cruz, M.C; Ronald Ferrera-Cerrato, R.; Haller, V.V.; Vázquez, R.R.; Linares, L. F. 2001. Adaptacion y seleccion de microorganismos autoctonos em Medios de cultivos enriquecidos con petroleo crudo. Agrociencia, 423-434.

Rivera-Cruz, M.C.; Trujillo-Narcía, A. 2004. Estudio de toxicidad vegetal em suelos com petróleos nuevo e intemperizado. Interciencia, 29(7): 369-376.

Systat Inc. 2002. SYSTAT 10.2 SYSTAT Software Inc.

Victorio, S.S.; Camargo, A.F.M.; Henry-Silva, G.G. 2006. Influência de diferentes concentrações de petróleo sobre macrófita aquática submersa enraizada Egeria densa. Livro de Resumos da Reunião Anual de Avaliação PRH-ANP 2006, 117-120.

Recebido em 10/03/2008

Aceito em 16/04/2009 